\title{
The symbolic efficacy of medicinal plants: practices, knowledge, and religious beliefs amongst the Nalu healers of Guinea-Bissau
}

\author{
Amélia Frazão-Moreira
}

\begin{abstract}
Background: In attempting to understand how the use of medicinal plants is symbolically valued and transformed according to specific cosmologies, we gain valuable insight into the ethnopharmacologial practices, in terms of the major role played by healers, as custodians of local ethnobotanical knowledge, but also as ritual masters.

Thus, the goal of this paper is to understand how medicinal plants are used differently depending on a combination between the healers' field of expertise and personal history on the one hand, and the diversified religious and symbolical frameworks on the other.

Methods: This essay is based on intense ethnographical research carried out amongst the Nalu people of Guinea-Bissau. Methods included participant observation and semi-directed interviews with six locally-renown healers (four men and two women). The progress of their work and the changes operated within the sets of beliefs associated with ethnopharmacological practices were registered by means of repeated field visits.

Results: A total of 98 species and 147 uses are accounted for, as well as a description of the plant parts that were used, as well as the methods of preparation and application according to the different healers' specialized practices. At the same time, this research describes those processes based on pre-Islamic and Muslim cosmologies through which medicinal plants are accorded their value, and treatments are granted their symbolic efficiency.

Conclusions: Medicinal plants are valued differently in the pre-Islamic medicine and in the medicine practiced by Islamic masters. The increasing relevance of Islam within this context has affected the symbolic framework of ethnopharmacological practices. Nevertheless, the endurance of those processes by which symbolic efficiency is attributed to local treatments based on plants is explained not only by the syncretic nature of African Islam, but also by the fact that patients adopt different therapeutic pathways simultaneously.
\end{abstract}

Keywords: Healers, Traditional medicine, Islam, Medicinal plants, Ethnopharmacological knowledge, Cosmologies, Guinea-Bissau, Africa

\section{Background}

In the West African rural contexts we can observe the daily collection and distribution of vegetable products for medicinal use. In poor countries, where public health systems are under-resourced, the practices of traditional medicine are of great importance. This is certainly the case of Guinea-Bissau [1, 2].

The Guinea-Bissau traditional medicinal systems are largely based on the use of plants for pharmacological

Correspondence: amoreira@fcsh.unl.pt

Centre for Research in Anthropology, Faculty of Social Sciences and

Humanities, Universidade Nova de Lisboa, Lisbon, Portugal use. Some studies on medicinal plants have been carried out in different ethnic groups, namely Fulani [3] and Bijagó $[4,5]$. However, those studies were mainly focused on botanical [6-9] or pharmacological analysis $[3,10,11]^{1}$.

This paper aims to present the uses of medicinal plants in the context of Nalu people (southern Guinea-Bissau) based on an ethnobotanical framework that focuses on the connection between plant uses and symbolic aspects. The proposed goal is to understand rationalities, cognitive processes and behaviors involving ethnopharmacological practices, while striving not to take local knowledge out of 
context, but instead to reflect on how it was generated, transformed, and connected to specialized practices and cosmological beliefs [12]. As Ingold suggests, local traditional knowledge is continually generated and regenerated within the contexts of people's skilled, practical involvement with significant components of the environment'; knowledge 'subsists in practical activities themselves, activities that may also be interpreted as ways of remembering' ([13], p. 307-8). In indigenous conceptualization, knowledge is not understood as 'a kind of substance' but rather as 'a kind of process'.

On the other hand, this paper places its main focus on the healers, as detainers of ethnopharmacological knowledge and guarantors of the population's survival. Thus, the main focus will be the subjects and their experience of the treatment and cure processes, rather than the medicinal plants per se.

Local treatment and cure processes include: cultural representations of health and illness; local healers' knowledge; nature and world-view perceptions shared by healers and patients; rituals and symbols that legitimize the efficacy of treatments. These elements must be taken together to understand the emic processual efficacy [14]. Said processes imply the coexistence of ancestral local practices and global dynamics of change. We can find different, complementary and simultaneous therapeutic practices in any African culture (e.g. [15-18]).

Based on these assumptions, the present work addresses two main questions: a) to what extent does the Nalu healers' use of the same plants vary according to their expertise and personal history; b) what role do cosmological perceptions and religious beliefs play in the use of medicinal plants.

Our goal is not providing a description of the ritual or magical uses of plants, but rather of the magical processes that confer symbolical efficacy on the medicinal plants which are not used in ritualized cures. Even though healers and patients alike share a common worldview, the precise and detailed features of said processes, or in other words, the specific actions performed by healers to endow the plants with symbolical efficacy, are in most cases not known to the patients.

This means that while a study focused on the plants and the observation of their therapeutic uses reveals two distinct types of plants used in the African medicinal systems - non ritual plants, used in non-religious treatments, and ritual plants used in ritualistic treatments (e.g. $[19,20])$, when we turn to the processes and practices through which healers confer efficacy on the plants, that distinction disappears. Thus, the working hypothesis for this paper is that, amongst the Nalu the distinction between the practical sphere comprised by the knowledge of certain plants' beneficial properties, and the magical sphere of supernatural intervention needed to render those properties effective, is meaningless from an emic point of view.

On the other hand, we argue that the means used to confer symbolical efficacy upon the treatments using medicinal plants, are different for Nalu healers who summon non-Muslim supernatural entities and those who invoke the words of the Quran.

\section{Population study}

The Nalu people inhabit Guinea-Bissau and Republic of Guinea. The community under analysis is located in Guinea-Bissau in the Cantanhez National Park (Fig. 1).

Nalu economy relies essentially on agriculture, more specifically on rice-growing in flooded areas and shifting cultivation, according to the traditional African slash-andburn system, as well as on the trade in fruit products and palm oil. Various ecosystems are identified locally as: forest (n'koi); new slash-and-burn field (kakoi); savannah (m’báké); mangrove (n’kim); rice-swamp (n'dále) and palm tree grove (n'búm) [21].

Similarly to other African social organizations, the ordering of productive and reproductive resources, and consequently political power, is mainly influenced by lineage. Together with kinship, distinctions of gender and principles of seniority are crucial to their social and economic organization. In terms of Religion, the Nalu of Cantanhez were Islamized at the beginning of the 20th century $[22,23]$, and while pre-Islamic beliefs and practices persisted among the Nalu until the turn of the 20th century, surviving in co-existence with Islamic practices until recently, currently they are in the process of being substituted altogether by Islamic-based practices.

\section{Methods}

The data presented was gathered in the course of a broader research, whose aim was to understand the process of the social appropriation of nature and plant classification [24]. An ethnographic methodology was used in this study. The procedures for gathering information on the medical practices and knowledge of the Nalu were participant observation and semi-directed interviews $[25,26]$. The present work is based on the information shared by six healers: two old women, two old men, and two younger men. For the most part, the information was obtained during plant-collecting in the forest and savannah, or during the preparation of medicines in the villages. All healers agreed, through prior verbal informed consent, to participate in the study and for their image to appear in scientific publications. The preliminary data collected were reported to a local NGO [27], and final data and later publications were returned to the community.

Initial fieldwork amounted to a total of 16 months, and was conducted throughout the period between 1993 


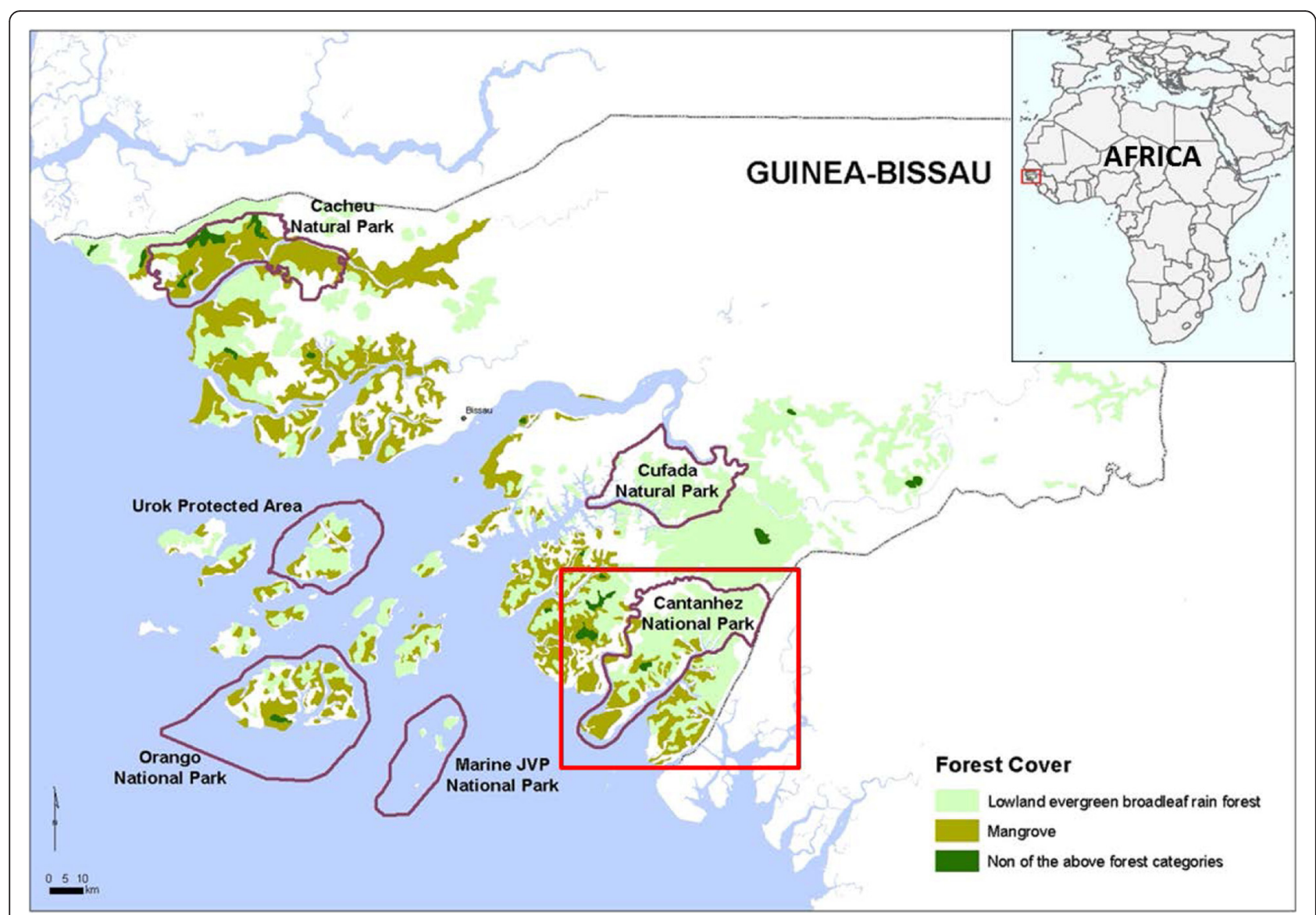

Fig. 1 Localization of Guinea-Bissau and the Catanhez National Park

and 1996. More recent field missions, in 2008, 2009, 2012 and 2013, made it possible to revisit the ethnographic setting and confirm the progress of the healers' work. Arafan Sané and Tcherno Camará, two of the well-reputed men healers in the region, have since passed away. In both cases, one of their male children - Iafai Sané and Amará Camará respectively - occupied their place, thus ensuring that at least part of their knowledge of medicinal plants will endure.

Herbarium vouchers were collected with informants, preserved according to the usual herbarium techniques, and the specimens deposited in the herbarium of IICT (Tropical Research Institute), Lisbon (LISC). The collection of data was made according to the national and international guidelines, with the support and agreement of local communities and Guinea-Bissau institutions (Iniciativa de Cantanhez and, after 2008, Instituto da Biodiversidade e das Áreas Protegidas). Identification of the specimens was based on a complete taxonomic study using Flora of West Tropical Africa [28] and through comparison with specimens already identified in the LISC Herbarium, which possesses the most important collection of specimens from the Guinea-Bissau. Plant names have been checked and updated through online data provided by the Royal Botanic Gardens, Kew and Missouri Botanical Garden (www.theplantlist.org).

The ethnopharmacological and ethnographic information was subjected to a qualitative analysis (e.g. [26, 29]).

\section{Results and discussion}

Local agents and perceptions of illnesses

Our observations enable us to assert that in the Nalu communities, different agents coexist in the treatment of different illnesses: healers who are specialized in the treatment of specific ailments, mostly physical disturbances, without resource to divination; djambakus (healers who are diviners and make ritualized treatments); marabouts (Muslim medicine-men); and even ordinary members of the social group, especially the elderly.

Despite the fact that any "classification of sicknesses" is constructed and reconstructed according to context, and that the links between nosological symptoms and categories - as between kinds of sickness and therapeutics -, are not reciprocal $[15,16,30]$, after systematizing local medical conceptions we can say that sicknesses fall essentially into two categories: "simple" sicknesses - manifestations 
of various physical symptoms-, and "irán sicknesses". Iráns are supernatural beings that populate the Nalu imagery, and irán diseases are disturbances, most of them of psychic nature, believed to be provoked by these spirits.

In the treatment and cure of the illnesses, different symbolic and cosmological frameworks are called forth, both pre-Islamic and Muslim beliefs. Healers summon the power of the spirits (iráns), which is essential to their cosmological explanation of the world and nature, whereas for Muslim medicine-men (marabouts) the effectiveness of the remedies resides basically in its association with the "sacred word" of the Quran.

Another important aspect derives from the fact that the infirm pursue different therapies and visit several agents of traditional medicine while also - and often simultaneously - seeking the help of state health services, based on the belief in the effectiveness of modern medicine ("the white man's medicine"), following the very same process that was theorized by authors who examined African medicine, such as Augé [15] and Fassin [16].

\section{Specialized knowledge of medicinal plants}

One hundred and forty seven different uses derived from ninety eight species were shared by healers. They are listed and ordered by healer and by the sicknesses treated by the plants used (Table 1 ).

There are certain medicinal plants known to all the Nalu including the children, such as n'fol (Sarcocephalus latifolius). But only a few amongst the elder members can identify the ailments that these plants can cure, and only healers know which parts of the plants must be used, as well as the specific combinations, preparation and dosing that apply in each particular circumstance.

The knowledge displayed by various healers is similar in terms of the number of medicinal specimens (23 plants on average), with the exception of Hajia Bintu Camará, who only mentioned eight plants. The treatments performed by Hajia Bintu, an elderly woman who had been a midwife, were essentially intended for the village members, especially pregnant women and children. Her medicinal practice relied on her skills and reputation as a midwife, having perfected herself in the prophylactic use of medicinal plants on pregnant women and new-born infants ${ }^{2}$.

In his turn, Usumane Cassamá was still a young man when we first started our investigation, still in the first stages of his practice as a healer. He had learnt the use of plants from his father, who had meanwhile passed away, and started being called upon to prepare medicines, mostly by his village relatives. In the course of the following years, his reputation grew. All the other healers - Arafan Sané, Dgibi Camará, Fatu Bangorá and Tcherno Camará -, were already celebrated healers who treated patients from different villages or even distant regions.
Learning the ability to heal using plants develops differently in each case. Some healers start by learning those medicinal uses of plants known as "household remedies", since they constitute the secret of a given lineage. The efficacy of these medicines is imbued with an ancestral symbolic significance, as will be shown below.

But there is also another way to obtain knowledge of medicinal plants: learning from other healers. Any individual, of any age or gender can seek a healer and ask to be apprenticed, which he/she may agree to in return for a fee, whether in money or goods. Healers who know certain household remedies can also increase their knowledge by learning new skills, thus increasing the number of diseases they can cure.

Thus, specialization derives from an individual's expertise in the treatment of specific diseases with the use of certain plants, in addition to possessing specific knowledge on the combination of vegetable ingredients to make medicines. As healer Arafan Sané would say: "All plants are medicinal. All plants are good for curing, we need to know them, some people know some plants, and other people know others".

Here are three examples of medicinal plants that, like many others, were used in various ways by different healers:

- n'fankok (Paullinia pinnata) used to treat "child weakness" (Fatu Bangorá), to fight scabies (Usumane Cassamé), and in the treatment of child diarrhea (Hajia Bintu Camará).

- n'tonkindjá (Ficus sur) used for lower back aches (Arafan Sané), failure to menstruate (Fatu Bangorá) and for stomach aches (Tcherno Camará e Dgibi Camará).

- n'fol (Sarcocephalus latifolius) used against intestinal parasites (Arafan Sané), constipation (Hajia Bintu Camará), stomach aches (Tcherno Camará e Dgibi Camará) and in the treatments that accompany pregnancy (Fatu Bangorá).

In terms of plant-picking habits, all healers follow a common principle: harvesting the vegetable elements needed for preparing medicine causing the least possible damage to the plants. Thus, while picking roots for instance, only a sufficient portion of the root is cut so as not to compromise the plant's survival, just as in the collection of tree bark, the least damaged barks are chosen. These practices are just as much in accordance with a practical concern with the preservation of natural resources, as with an ontological respect for non-human beings, providing a basis for sustainability and contributing to the preservation of biodiversity.

The collection of the medicinal plants used by Tcherno Camará and Dgibi Camará was carried out together with 
Table 1 Plant used by healers for medicinal purposes

\begin{tabular}{|c|c|c|c|c|c|c|c|c|}
\hline Family & Genus and species & Nalu name & Voucher $^{a}$ & Healer $^{\mathrm{b}}$ & Ailment & $\begin{array}{l}\text { Part of } \\
\text { plant used }\end{array}$ & Preparation & Application \\
\hline \multirow[t]{5}{*}{ Acanthaceae } & $\begin{array}{l}\text { Asystasia gangetica (L.) } \\
\text { T.Anderson }\end{array}$ & & Moreira 85 & UC & open wounds & bark & scrape & $\overline{\text { poultice }}$ \\
\hline & Anacardium occidentale L. & ialikê & Moreira 213 & $\mathrm{TC} / \mathrm{DC}$ & lower back pain & leaves & none & topical \\
\hline & Mangifera indica L. & n'mango & Moreira 32 & $\mathrm{TC} / \mathrm{DC}$ & scabies & leaves & macerate & topical \\
\hline & $\begin{array}{l}\text { Sorindeia juglandifolia } \\
\text { (A.Rich.) Planch. ex Oliv. }\end{array}$ & n'txaluas & Moreira 154 & $\mathrm{FB}$ & $\begin{array}{l}\text { children's } \\
\text { disease }\end{array}$ & leaves & macerate & drink \\
\hline & Spondias mombin $\mathrm{L}$. & n'fal & Moreira 24 & $\mathrm{TC} / \mathrm{DC}$ & eye pains & leaves & macerate & wash \\
\hline Anisophylleaceae & $\begin{array}{l}\text { Anisophyllea laurina } \\
\text { R.Br. ex Sabine }\end{array}$ & n'sunt & Moreira 57 & $\mathrm{TC} / \mathrm{DC}$ & eye pain & leaves & moisten & topical \\
\hline \multirow[t]{5}{*}{ Annonaceae } & Annona glabra $\mathrm{L}$. & n'bonhé & Moreira 38 & $\mathrm{FB}$ & stomach ache & root & $\begin{array}{l}\text { infusion or } \\
\text { macerate }\end{array}$ & drink \\
\hline & Annona muricata $\mathrm{L}$. & n'sóp sóp & Moreira 73 & DC & stomach ache & leaves & boil & drink \\
\hline & Uvaria chamae P.Beauv. & n'pinden & Moreira 155 & UC & haemorrhoids & root & boil & drink \\
\hline & & & & AS & open wound & leaves & triturate & topical \\
\hline & $\begin{array}{l}\text { Xylopia aethiopica } \\
\text { (Dunal) A. Rich. }\end{array}$ & n'sél & Moreira 168 & $\mathrm{FB}$ & stomach ache & fruit & boil & drink \\
\hline \multirow[t]{15}{*}{ Apocynaceae } & Alstonia congensis Engl. & ianke & Moreira 27 & $\mathrm{TC} / \mathrm{DC}$ & fatigue & bark & macerate & drink \\
\hline & & & & & swelling & latex & none & topical \\
\hline & Cf. Strophanthus hispidus DC. & n'fam & Moreira 78 & UC & haemorrhoids & root & peel & swallow \\
\hline & & & & & $\begin{array}{l}\text { intestinal } \\
\text { parasites }\end{array}$ & root & peel & swallow \\
\hline & Holarrhena floribunda & metxel & Moreira 86 & UC & stomach ache & bark & boil & drink \\
\hline & (G.Don) T.Durand \& Schinz & & & & venereal disease & latex & scrape & swallow \\
\hline & $\begin{array}{l}\text { Landolphia dulcis (Sabine } \\
\text { ex G.Don) Pichon }\end{array}$ & urém & Moreira 176 & $F B$ & pregnancy & leaves & macerate & drink \\
\hline & Landolphia heudelotii A.DC. & m’bolé & Moreira 158 & AS & diarrhoea & leaves & boil & drink \\
\hline & & & & & swelling & leaves & boil & drink \\
\hline & & & & $F B$ & swollen limbs & leaves & boil & drink \\
\hline & & & & UC & itching & leaves & boil & wash \\
\hline & Rauvolfia vomitoria Afzel. & n'ti kambirás & Moreira 14 & $\mathrm{TC} / \mathrm{DC}$ & impotence & stem & boil & drink \\
\hline & & & & & stomach ache & root & boil & drink \\
\hline & $\begin{array}{l}\text { Saba senegalensis (A.DC.) } \\
\text { Pichon }\end{array}$ & n'badak & Moreira 64 & $\mathrm{TC} / \mathrm{DC}$ & fever & leaves & infusion & wash \\
\hline & $\begin{array}{l}\text { Tabernaemontana africana } \\
\text { Hook. }\end{array}$ & n'lat laté & Moreira 15 & $\mathrm{TC} / \mathrm{DC}$ & venereal disease & stem & macerate & drink \\
\hline \multirow[t]{2}{*}{ Araceae } & $\begin{array}{l}\text { Anchomanes difformis } \\
\text { (Blume) Engl. }\end{array}$ & n'denkamdudu & Moreira 286 & AS & infertility & rhizome & boil & drink \\
\hline & Cercestis afzelii Schott & mandonha & Moreira 92 & UC & impotence & leaves & boil & drink \\
\hline Cannabaceae & Trema orientalis (L.) Blume & n'robta kabafar & Moreira 114 & AS & cough & bark & none & swallow \\
\hline \multirow[t]{3}{*}{ Capparaceae } & Capparis erythrocarpos Isert & néeu & Moreira 89, 120 & AS & headache & root & triturate & topical \\
\hline & & & & UC & headache & root & scrape & topical \\
\hline & $\begin{array}{l}\text { Maerua duchesnei } \\
\text { (De Wild.) F.White }\end{array}$ & maéf & Moreira 90, 314 & $\begin{array}{l}\text { AS } \\
\text { UC }\end{array}$ & $\begin{array}{l}\text { lower back pain } \\
\text { sprains }\end{array}$ & $\begin{array}{l}\text { leaves } \\
\text { leaves }\end{array}$ & $\begin{array}{l}\text { triturate } \\
\text { triturate }\end{array}$ & $\begin{array}{l}\text { poultice } \\
\text { poultice }\end{array}$ \\
\hline
\end{tabular}


Table 1 Plant used by healers for medicinal purposes (Continued)

\begin{tabular}{|c|c|c|c|c|c|c|c|c|}
\hline Caricaceae & Carica papaya $\mathrm{L}$. & n'pápa & Moreira 22 & $\mathrm{TC} / \mathrm{DC}$ & $\begin{array}{l}\text { risk of } \\
\text { miscarriage }\end{array}$ & root & boil & drink \\
\hline Celastraceae & Salacia senegalensis (Lam.) DC. & mankidés & Moreira 6 & $\mathrm{TC} / \mathrm{DC}$ & tooth decay & root & triturate & topical \\
\hline \multirow[t]{3}{*}{ Chrysobalanaceae } & Parinari excelsa Sabine & n'lut & Moreira 145, 196 & $F B$ & stomach ache & bark & boil & drink \\
\hline & & & & $\mathrm{TC} / \mathrm{DC}$ & cough & bark & macerate & swallow \\
\hline & & & & & open wound & bark & triturate & topical \\
\hline \multirow[t]{6}{*}{ Combretaceae } & Combretum micranthum G.Don & n'babass & Moreira 81, 200 & $\mathrm{TC} / \mathrm{DC}$ & scabies & root & triturate & topical \\
\hline & & & & & diabetes & leaves & boil & drink \\
\hline & & & & UC & scabies & root & scrape & topical \\
\hline & & & & & fever & leaves & boil & drink \\
\hline & Guiera senegalensis J.F.Gmel. & manáf náfen & Moreira 173 & AS & open wound & leaves & triturate & poultice \\
\hline & $\begin{array}{l}\text { Terminalia macroptera Guill. } \\
\text { \& Perr. }\end{array}$ & n'kone & Moreira 126 & AS & chest pain & leaves & boil & $\begin{array}{l}\text { drink and } \\
\text { wash }\end{array}$ \\
\hline \multirow[t]{2}{*}{ Compositae } & $\begin{array}{l}\text { Aedesia glabra (Klatt) } \\
\text { O.Hoffm. }\end{array}$ & n'tonpin-na & Moreira 262 & AS & $\begin{array}{l}\text { stomach and } \\
\text { body aches }\end{array}$ & root & boil & drink \\
\hline & $\begin{array}{l}\text { Gymnanthemum coloratum } \\
\text { (Willd.) H.Rob. \& B.Kahn }\end{array}$ & n'konkone & Moreira 95 & UC & scabies & leaves & macerate & wash \\
\hline \multirow[t]{2}{*}{ Connaraceae } & $\begin{array}{l}\text { Agelaea pentagyna (Lam.) } \\
\text { Baill. }\end{array}$ & & Moreira 190 & $\mathrm{HBC}$ & scabies & leaves & none & ointment \\
\hline & Cnestis ferruginea Vahl ex DC. & n'xéte nembel & Moreira 16 & $\mathrm{TC} / \mathrm{DC}$ & headache & leaves & macerate & wash \\
\hline \multirow[t]{2}{*}{ Costaceae } & Costus afer Ker Gawl. & mabôbé & Moreira 93, 122 & UC & eye pain & stem & $\begin{array}{l}\text { warm and } \\
\text { squeeze }\end{array}$ & topical \\
\hline & & & & AS & eye pain & stem & $\begin{array}{l}\text { warm and } \\
\text { squeeze }\end{array}$ & topical \\
\hline Crassulaceae & $\begin{array}{l}\text { Kalanchoe crenata (Andrews) } \\
\text { Haw. }\end{array}$ & n'txatxatxé & Moreira 242 & $\mathrm{HBC}$ & $\begin{array}{l}\text { newborn baby's } \\
\text { navel }\end{array}$ & leaves & $\begin{array}{l}\text { moisten } \\
\text { and } \\
\text { squeeze }\end{array}$ & topical \\
\hline \multirow[t]{2}{*}{ Cyperaceae } & Cyperus articulatus L. & n'téd & Moreira 121 & AS & $\begin{array}{l}\text { newborn with a } \\
\text { cough and } \\
\text { stomach ache }\end{array}$ & root & $\begin{array}{l}\text { triturate } \\
\text { and } \\
\text { macerate }\end{array}$ & drink \\
\hline & Scleria racemosa Poir. & $\begin{array}{l}\text { n'txapen ka } \\
\text { basop }\end{array}$ & Moreira 94 & UC & pregnancy & leaves & boil & drink \\
\hline Ebenaceae & Diospyros heudelotii Hiern & n'txambortá & Moreira 76 & DC & swollen limbs & leaves & macerate & $\begin{array}{c}\text { drink and } \\
\text { wash }\end{array}$ \\
\hline \multirow[t]{4}{*}{ Euphorbiaceae } & $\begin{array}{l}\text { Alchornea cordifolia (Schumach. } \\
\text { \& Thonn.) Müll.Arg. }\end{array}$ & n'sum-na & Moreira 4, 129 & AS & $\begin{array}{l}\text { chest ache } \\
\text { intestinal } \\
\text { parasites }\end{array}$ & $\begin{array}{l}\text { stem } \\
\text { root }\end{array}$ & $\begin{array}{l}\text { peel } \\
\text { boil }\end{array}$ & $\begin{array}{l}\text { wash } \\
\text { drink }\end{array}$ \\
\hline & & & & & $\begin{array}{l}\text { risk of } \\
\text { miscarriage }\end{array}$ & root & boil & drink \\
\hline & & & & $\mathrm{TC} / \mathrm{DC}$ & stomach ache & leaves & macerate & drink \\
\hline & Manihot esculenta Crantz & n'mandiok & Moreira 23 & $\mathrm{TC} / \mathrm{DC}$ & eye complaints & leaves & macerate & wash \\
\hline Hypericaceae & $\begin{array}{l}\text { Psorospermum glaberrimum } \\
\text { Hochr. }\end{array}$ & & Moreira 124 & AS & body aches & leaves & boil & drink \\
\hline Icacinaceae & $\begin{array}{l}\text { Icacina oliviformis (Poiret) } \\
\text { J.Raynal }\end{array}$ & n'putmé & Moreira 162 & $F B$ & children's disease & leaves & boil & drink \\
\hline Lamiaceae & $\begin{array}{l}\text { Clerodendrum splendens } \\
\text { G.Don }\end{array}$ & manar balé & Moreira 48 & $\mathrm{TC} / \mathrm{DC}$ & $\begin{array}{l}\text { snake bite } \\
\text { contraception }\end{array}$ & $\begin{array}{c}\text { root } \\
\text { bark and } \\
\text { stem }\end{array}$ & $\begin{array}{l}\text { macerate } \\
\text { none }\end{array}$ & $\begin{array}{l}\text { swallow } \\
\text { drink }\end{array}$ \\
\hline
\end{tabular}


Table 1 Plant used by healers for medicinal purposes (Continued)

\begin{tabular}{|c|c|c|c|c|c|c|c|c|}
\hline & Premna hispida Benth. & n'lum bafai & Moreira 68, & $\mathrm{HBC}$ & pregnancy & leaves & boil & drink \\
\hline & & & 110,170 & DC & haemorrhoids & leaves & macerate & drink \\
\hline & & & & $F B$ & haemorrhoids & leaves & $\begin{array}{l}\text { boil or } \\
\text { macerate }\end{array}$ & drink \\
\hline & Vitex madiensis Oliv. & n'sokór & Moreira 177 & $\mathrm{FB}$ & pregnancy & leaves & macerate & drink \\
\hline \multirow[t]{23}{*}{ Leguminosae } & Albizia zygia (DC.) J.F.Macbr. & masanp n'buk & Moreira 172 & $F B$ & palpitations & leaves & triturate & swallow \\
\hline & \multirow[t]{3}{*}{ Bauhinia thonningii Schum. } & \multirow[t]{3}{*}{ n'bukui } & \multirow[t]{3}{*}{ Moreira 30, 105} & \multirow[t]{2}{*}{$\mathrm{TC} / \mathrm{DC}$} & diarrhoea & leaves & $\begin{array}{l}\text { triturate } \\
\text { and } \\
\text { macerate }\end{array}$ & drink \\
\hline & & & & & haemorrhoids & bark & boil & drink \\
\hline & & & & UC & haemorrhoids & bark & boil & drink \\
\hline & \multirow[t]{2}{*}{$\begin{array}{l}\text { Caesalpinia benthamiana } \\
\text { (Baill.) Herend. \& Zarucchi }\end{array}$} & \multirow[t]{2}{*}{ n'pinkid sé } & \multirow[t]{2}{*}{ Moreira 51, 119} & AS & eye complaints & leaves & macerate & topical \\
\hline & & & & $\mathrm{TC} / \mathrm{DC}$ & open wound & $\begin{array}{l}\text { root and } \\
\text { leaves }\end{array}$ & triturate & topical \\
\hline & \multirow[t]{3}{*}{ Cassia sieberiana DC. } & \multirow[t]{3}{*}{ n'sansan } & \multirow[t]{3}{*}{ Moreira 33} & AS & body aches & root & macerate & drink \\
\hline & & & & \multirow[t]{2}{*}{$\mathrm{TC} / \mathrm{DC}$} & body aches & root & macerate & drink \\
\hline & & & & & impotence & root & macerate & drink \\
\hline & Cf. Abrus precatorius L. & & Moreira 83 & UC & impotence & leaves & none & swallow \\
\hline & $\begin{array}{l}\text { Desmodium velutinum (Willd.) } \\
\text { DC. }\end{array}$ & rap rap & Moreira 21 & $\mathrm{TC} / \mathrm{DC}$ & cholera & $\begin{array}{l}\text { leaves } \\
\text { and root }\end{array}$ & macerate & drink \\
\hline & $\begin{array}{l}\text { Detarium microcarpum Guill. } \\
\& \text { Perr. }\end{array}$ & m’béta & Moreira 63 & $\mathrm{TC} / \mathrm{DC}$ & uncertain & bark & macerate & wash \\
\hline & Dialium guineense Willd. & n'bim & Moreira 52 & $\mathrm{TC} / \mathrm{DC}$ & measles & leaves & boil & drink \\
\hline & \multirow{2}{*}{$\begin{array}{l}\text { Dichrostachys cinerea (L.) } \\
\text { Wight \& Arn. }\end{array}$} & \multirow[t]{2}{*}{ n'pinkid úne } & \multirow[t]{2}{*}{ Moreira 46, 96} & $\mathrm{TC} / \mathrm{DC}$ & headache & bark & peel & topical \\
\hline & & & & UC & bone pains & bark & peel & topical \\
\hline & \multirow[t]{3}{*}{ Erythrina senegalensis DC. } & \multirow[t]{3}{*}{ n'txákarfatch } & \multirow{3}{*}{$\begin{array}{l}\text { Moreira 47, } \\
175\end{array}$} & $\mathrm{FB}$ & sore throat & bark & boil & drink \\
\hline & & & & & palpitations & root & $\begin{array}{l}\text { peel and } \\
\text { macerate }\end{array}$ & drink \\
\hline & & & & $\mathrm{TC} / \mathrm{DC}$ & pregnancy & bark & $\begin{array}{l}\text { peel and } \\
\text { boil }\end{array}$ & drink \\
\hline & \multirow{3}{*}{$\begin{array}{l}\text { Parkia biglobosa (Jacq.) } \\
\text { G.Don }\end{array}$} & \multirow[t]{3}{*}{ n'iú } & \multirow[t]{3}{*}{ Moreira 164} & $\mathrm{FB}$ & treponematoses & leaves & triturate & poultice \\
\hline & & & & & rheumatism & leaves & triturate & poultice \\
\hline & & & & $\mathrm{TC} / \mathrm{DC}$ & stomach ache & bark & boil & drink \\
\hline & Pterocarpus erinaceus Poir. & n'sia & Moreira 9 & $\mathrm{TC} / \mathrm{DC}$ & cough, chest ache & bark & boil & drink \\
\hline & $\begin{array}{l}\text { Samanea dinklagei (Harms) } \\
\text { Keay }\end{array}$ & masamp & Moreira 152 & DC & lower back pain & root & boil & $\begin{array}{l}\text { drink and } \\
\text { wash }\end{array}$ \\
\hline Loganiaceae & Usteria guineensis Willd. & n'átá uóké & Moreira 293 & AS & lower back pain & leaves & triturate & poultice \\
\hline \multirow[t]{5}{*}{ Malvaceae } & Ceiba pentandra (L.) Gaertn. & n'kauuê & Moreira 59 & $\mathrm{TC} / \mathrm{DC}$ & open wound & bark & triturate & topical \\
\hline & $\begin{array}{l}\text { Cola nitida (Vent.) Schott. \& } \\
\text { Endl. }\end{array}$ & n'kola & Moreira 72 & DC & venereal disease & leaves & $\begin{array}{c}\text { burn and } \\
\text { boil }\end{array}$ & $\begin{array}{l}\text { topical and } \\
\text { wash }\end{array}$ \\
\hline & \multirow{2}{*}{$\begin{array}{l}\text { Hibiscus sterculiifolius (Guill. \& } \\
\text { Perr.) Steud. }\end{array}$} & \multirow[t]{2}{*}{ n'fakaf } & \multirow[t]{2}{*}{ Moreira 75} & \multirow[t]{2}{*}{ DC } & influenza & leaves & macerate & drink \\
\hline & & & & & lice & leaves & macerate & wash \\
\hline & Sida acuta Burm.f. & n'téksen & Moreira 10 & $\mathrm{TC} / \mathrm{DC}$ & lice & leaves & macerate & wash \\
\hline Menispermaceae & $\begin{array}{l}\text { Cissampelos mucronata } \\
\text { A.Rich. }\end{array}$ & n'néo fáfak & Moreira 40 & $\mathrm{TC} / \mathrm{DC}$ & stomach ache & root & macerate & drink \\
\hline
\end{tabular}


Table 1 Plant used by healers for medicinal purposes (Continued)

\begin{tabular}{|c|c|c|c|c|c|c|c|c|}
\hline & Triclisia patens Oliv. & $\begin{array}{l}\text { manar } \\
\text { kambantxô }\end{array}$ & Moreira 132 & $\mathrm{HBC}$ & $\begin{array}{l}\text { expectant } \\
\text { mother's lack of } \\
\text { milk }\end{array}$ & leaves & macerate & drink \\
\hline \multirow[t]{6}{*}{ Moraceae } & Ficus exasperata Vahl & n'txéf & Moreira 197 & $\mathrm{TC} / \mathrm{DC}$ & impotence & leaves & macerate & drink \\
\hline & \multirow[t]{2}{*}{ Ficus aff. umbellata Vahl } & \multirow[t]{2}{*}{ n'fór } & \multirow[t]{2}{*}{ Moreira 84} & $\mathrm{TC} / \mathrm{DC}$ & toothache & stem & scrape & topical \\
\hline & & & & UC & lower back pain & leaves & triturate & topical \\
\hline & \multirow[t]{3}{*}{ Ficus sur Forssk. } & \multirow[t]{3}{*}{ n'tonkindjá } & \multirow[t]{3}{*}{ Moreira 128, 131} & AS & lower back ache & root & $\begin{array}{l}\text { triturate } \\
\text { and boil }\end{array}$ & poultice \\
\hline & & & & $F B$ & $\begin{array}{l}\text { failure to } \\
\text { menstruate }\end{array}$ & root & macerate & drink \\
\hline & & & & $\mathrm{TC} / \mathrm{DC}$ & stomach ache & bark & macerate & drink \\
\hline Myrtaceae & Psidium guajava $\mathrm{L}$. & goiaba & Moreira 66 & $\mathrm{TC} / \mathrm{DC}$ & dysentery & leaves & boil & drink \\
\hline Passifloraceae & $\begin{array}{l}\text { Smeathmannia pubescens } \\
\text { Sol. ex R.Br. }\end{array}$ & n'baptame & Moreira 337 & AS & $\begin{array}{l}\text { stomach ache } \\
\text { venereal disease }\end{array}$ & $\begin{array}{l}\text { root } \\
\text { root }\end{array}$ & $\begin{array}{l}\text { boil } \\
\text { boil }\end{array}$ & $\begin{array}{l}\text { drink } \\
\text { drink }\end{array}$ \\
\hline \multirow[t]{6}{*}{ Phyllanthaceae } & \multirow[t]{2}{*}{$\begin{array}{l}\text { Bridelia micrantha (Hochst.) } \\
\text { Baill. }\end{array}$} & \multirow[t]{2}{*}{ n'tak } & \multirow[t]{2}{*}{ Moreira 171, 261} & AS & stomach ache & root & boil & drink \\
\hline & & & & $\mathrm{FB}$ & haemorrhoids & leaves & $\begin{array}{l}\text { triturate } \\
\text { and } \\
\text { macerate }\end{array}$ & drink \\
\hline & \multirow[t]{3}{*}{ Hymenocardia acida Tul. } & \multirow[t]{3}{*}{ matik sé } & \multirow[t]{3}{*}{ Moreira 166} & $F B$ & treponematoses & leaves & paste & poultice \\
\hline & & & & & rheumatism & leaves & paste & poultice \\
\hline & & & & $\mathrm{TC} / \mathrm{DC}$ & $\begin{array}{l}\text { temporary } \\
\text { blindness }\end{array}$ & leaves & macerate & wash \\
\hline & $\begin{array}{l}\text { Phyllanthus muellerianus } \\
\text { (Kuntze) Exell }\end{array}$ & mafér & Moreira 148 & $F B$ & pregnancy & leaves & boil & drink \\
\hline \multirow[t]{2}{*}{ Plantaginaceae } & \multirow[t]{2}{*}{ Scoparia dulcis L. } & \multirow[t]{2}{*}{ n'txinké } & \multirow[t]{2}{*}{ Moreira 109} & \multirow[t]{2}{*}{ UC } & eye pain & leaves & macerate & topical \\
\hline & & & & & $\begin{array}{l}\text { children's } \\
\text { stomach ache }\end{array}$ & leaves & boil & drink \\
\hline Poaceae & $\begin{array}{l}\text { Oxytenanthera abyssinica } \\
\text { (A.Rich.) Munro }\end{array}$ & n'fon & Moreira 87 & UC & $\begin{array}{l}\text { abortion } \\
\text { post-natal } \\
\text { problems }\end{array}$ & $\begin{array}{l}\text { leaves } \\
\text { leaves }\end{array}$ & $\begin{array}{l}\text { boil } \\
\text { boil }\end{array}$ & $\begin{array}{l}\text { drink } \\
\text { drink }\end{array}$ \\
\hline \multirow[t]{12}{*}{ Rubiaceae } & \multirow[t]{2}{*}{ Aidia genipiflora (DC.) Dandy } & \multirow[t]{5}{*}{ n'armass } & \multirow[t]{2}{*}{ Moreira 335} & \multirow[t]{2}{*}{ AS } & impurity & root & foam & wash \\
\hline & & & & & pregnancy & leaves & macerate & drink \\
\hline & \multirow[t]{2}{*}{ Canthium sp. } & & Moreira 165 & $\mathrm{FB}$ & treponematoses & leaves & paste & poultice \\
\hline & & & & & rheumatism & leaves & paste & poultice \\
\hline & $\begin{array}{l}\text { Crossopteryx febrifuga (Afzel. } \\
\text { ex G.Don) Benth. }\end{array}$ & & Moreira 174 & $\mathrm{FB}$ & pregnancy & leaves & macerate & drink \\
\hline & $\begin{array}{l}\text { Gardenia ternifolia subsp. } \\
\text { jovis-tonantis (Welw.) Verdc. } \\
\text { var. jovis tonantis }\end{array}$ & n'dué & Moreira 125 & AS & impurities & root & foam & wash \\
\hline & Morinda chrysorhiza (Thonn.) & n'tunké & Moreira 118 & AS & post-natal & leaves & boil & drink \\
\hline & & & & $\mathrm{TC} / \mathrm{DC}$ & post-natal & leaves & boil & wash \\
\hline & $\begin{array}{l}\text { Morinda morindoides (Baker) } \\
\text { Milne-Redh. }\end{array}$ & $\begin{array}{l}\text { n'txéf kam } \\
\text { nhalankôk }\end{array}$ & Moreira 113 & AS & body aches & leaves & boil & fumigate \\
\hline & $\begin{array}{l}\text { Pavetta corymbosa (DC.) } \\
\text { F.N.Williams }\end{array}$ & & Moreira 269 & $\mathrm{HBC}$ & open wound & bark & $\begin{array}{l}\text { peel and } \\
\text { triturate }\end{array}$ & topical \\
\hline & $\begin{array}{l}\text { Psychotria peduncularis } \\
\text { (Salisb.) Steyerm. }\end{array}$ & n'tokoi & Moreira 161 & AS & open wound & leaves & heat & topical \\
\hline & & & & $\mathrm{FB}$ & children's disease & leaves & boil & drink \\
\hline
\end{tabular}


Table 1 Plant used by healers for medicinal purposes (Continued)

\begin{tabular}{|c|c|c|c|c|c|c|c|c|}
\hline & $\begin{array}{l}\text { Sarcocephalus latifolius (Sm.) } \\
\text { E.A.Bruce }\end{array}$ & $n^{\prime}$ fol & Moreira 82 & AS & $\begin{array}{l}\text { intestinal } \\
\text { parasites }\end{array}$ & root & $\begin{array}{l}\text { scrape and } \\
\text { macerate }\end{array}$ & drink \\
\hline & & & & $\mathrm{HBC}$ & constipation & leaves & boil & drink \\
\hline & & & & $\mathrm{TC} / \mathrm{DC}$ & stomach ache & root & $\begin{array}{l}\text { peel and } \\
\text { macerate }\end{array}$ & drink \\
\hline & & & & UC & pregnancy & leaves & macerate & drink \\
\hline \multirow[t]{5}{*}{ Rutaceae } & Citrus limon (L.) Osbeck & n'sinim nelbéne & Moreira 67 & $\mathrm{TC} / \mathrm{DC}$ & scabies & leaves & boil & wash \\
\hline & & & & & venereal disease & $\begin{array}{l}\text { leaves } \\
\text { and root }\end{array}$ & boil & drink \\
\hline & & & & & $\begin{array}{l}\text { chronic open } \\
\text { wound }\end{array}$ & fruit & moisten & topical \\
\hline & & & & & haemorrhoids & fruit & boil & drink \\
\hline & $\begin{array}{l}\text { Zanthoxylum leprieurii Guill. \& } \\
\text { Perr. }\end{array}$ & mabár & Moreira 104 & UC & $\begin{array}{l}\text { risk of } \\
\text { miscarriage }\end{array}$ & root & $\begin{array}{l}\text { peel and } \\
\text { macerate }\end{array}$ & drink \\
\hline \multirow[t]{7}{*}{ Sapindaceae } & Allophylus africanus P.Beauv. & & Moreira 160 & $\mathrm{HBC}$ & pregnancy & leaves & boil & drink \\
\hline & & & & $\mathrm{FB}$ & swollen limbs & leaves & macerate & $\begin{array}{l}\text { drink and } \\
\text { wash }\end{array}$ \\
\hline & $\begin{array}{l}\text { Lecaniodiscus cupanioides } \\
\text { (Planch.) ex Benth. }\end{array}$ & n'sonran & Moreira 80 & UC & cough & root & $\begin{array}{c}\text { peel, } \\
\text { triturate } \\
\text { and } \\
\text { macerate }\end{array}$ & drink \\
\hline & & & & & hoarseness & root & $\begin{array}{c}\text { peel, } \\
\text { triturate } \\
\text { and } \\
\text { macerate }\end{array}$ & drink \\
\hline & Paullinia pinnata L. & n'fankok & Moreira 76 & $\mathrm{HBC}$ & $\begin{array}{l}\text { diarrhoea in } \\
\text { children }\end{array}$ & leaves & macerate & drink \\
\hline & & & & $\mathrm{FB}$ & $\begin{array}{l}\text { weakness in } \\
\text { children }\end{array}$ & leaves & macerate & $\begin{array}{l}\text { drink and } \\
\text { wash }\end{array}$ \\
\hline & & & & UC & scabies & leaves & boil & wash \\
\hline Smilacaceae & Smilax anceps Willd. & $\begin{array}{l}\text { n'pôrkam } \\
\text { nunpun }\end{array}$ & Moreira 298 & AS & body aches & root & boil & drink \\
\hline Thymelaeaceae & Dicranolepis disticha Planch. & n'saldendek & Moreira 97 & UC & $\begin{array}{l}\text { intestinal } \\
\text { parasites }\end{array}$ & root & $\begin{array}{c}\text { peel, } \\
\text { triturate } \\
\text { and } \\
\text { macerate }\end{array}$ & drink \\
\hline Vitaceae & Cissus rufescens Guill. \& Perr. & & Moreira 232 & $\mathrm{FB}$ & swollen limbs & $\begin{array}{l}\text { leaves } \\
\text { and root }\end{array}$ & $\begin{array}{l}\text { peel and } \\
\text { triturate }\end{array}$ & topical \\
\hline Zingiberaceae & $\begin{array}{l}\text { Aframomum alboviolaceum } \\
\text { (Ridl.) K.Schum. }\end{array}$ & mabôbé & Moreira 115 & AS & stomach ache & root & boil & drink \\
\hline \multirow[t]{5}{*}{ Unidentified } & & $\begin{array}{l}\text { mabobese } \\
\text { tchill }\end{array}$ & Moreira 74 & $D C$ & $\begin{array}{l}\text { children's } \\
\text { disease }\end{array}$ & $\begin{array}{l}\text { root and } \\
\text { leaves }\end{array}$ & $\begin{array}{l}\text { boil and } \\
\text { paste }\end{array}$ & $\begin{array}{l}\text { drink and } \\
\text { poultice }\end{array}$ \\
\hline & & méné & Moreira 41 & $\mathrm{TC} / \mathrm{DC}$ & swollen limbs & leaves & boil & $\begin{array}{l}\text { wash and } \\
\text { fumigate }\end{array}$ \\
\hline & & n'pitió & Moreira 28 & $\mathrm{TC} / \mathrm{DC}$ & dental caries & bark & triturate & topical \\
\hline & & n'timé & Moreira 153 & $\mathrm{FB}$ & children's disease & leaves & macerate & drink \\
\hline & & olo & Moreira 35 & $\mathrm{TC} / \mathrm{DC}$ & $\begin{array}{l}\text { pulmonary } \\
\text { complaints }\end{array}$ & leaves & macerate & drink \\
\hline
\end{tabular}

\footnotetext{
a Voucher: plant specimens are deposited in LISC Herbarium (IICT -Tropical Research Institute, Lisbon)

${ }^{b}$ Healer: AS Arafan Sane, DC Dgibi Camará, FB Fatu Bangorá, HBC Hajia Bintu Camará, TC Tcherno Camará, TC/DC Tcherno Camará and Dgibi Camará, UC Usumane Cassamá
} 
both healers. However, as it will soon become clear, the symbolical proceedings involved in the treatments used by each of these two healers were totally different, since Tcherno Camará relied on pre-Islamic symbolical elements while Dgibi Camará is a marabout and thus followed Muslim practices.

We can therefore claim to have found two kinds of specialization; one specialization consisting in the knowledge of the medicinal characteristics of plants, and the another in the symbolic efficiency of the treatments that implied the use of plants.

\section{Protection mothers and children - healer Fatu Bangorá}

The healer Fatu Bangorá is an old woman who specialized in assisting pregnant women and curing children's diseases, besides treating a large number of adult ailments. She learned some of these medicines from her mother, but there was a specific episode that drew her to this activity. Her mother became gravely ill, and a healer from different ethnic group (Sussu), who lived the Republic of Guinea and was visiting the region, was the only one to hit upon the right treatment. When she saw this, Fatu decided to "buy his medicines", or in other words to learn from him in exchange for payment in goods. From that moment on, she has been collecting different knowledge on medicinal plants from many other healers, not the least because, as she explained, she "had many children and sickness can fall upon us unexpectedly".

She uses a broad range of plants (Table 1). One example concerns the prevention of the illness called foie kumbé ("big wind"). In this situation, women "expel blood during pregnancy and later die during childbirth, along with the infant" (very likely corresponding to situations in which the placenta is not securely attached). She uses the leaves of mékinha (Crossopteryx febrifuga), n'sansan (Cassia sieberiana), n'sokor (Vitex madiensis) and urém (Landolphia dulcis), dried and macerated in cold water. The resulting liquid is drunk by the women daily, starting in the third month of pregnancy.

When the baby is born, it is washed with the same medicine and also begins to drink it. When the breastfeeding period ends, the mother takes the child to the house of the healer, who will proceed with a symbolic ceremony. In this so-called "law of the remedy", Fatu cuts off a small lock of the child's hair, thus marking the end of the preventive period. As an expert in the treatment of childhood diseases, the pregnant mothers assisted by her, essentially make use of her services when their children become ill.

Despite not belonging to a lineage of traditional medicine experts, this healer gained recognition. The means by which she acquired knowledge of medicinal plants was not a ritual learning, neither was it based on any kind of magical initiation. Nevertheless, there are symbolical elements connected with some of the treatments, and they are marked by ritualistic practices, as in the example shown.

\section{The symbolic efficacy of mesinhos di casa - healers Arafan Sané and Tcherno Camará}

Some healers anticipate and cure with mesinhos di casa ("household remedies") - secrets about the use of certain plants that are passed down from parents to children, from grandparents to grandchildren. Arafan Sané and Tcherno Camará, now deceased, were two such healers. As such, they shared their knowledge of medicinal plants, but only within the boundaries of what they considered not to have a secret character. In spite of this, after several months of fieldwork, they explained the ritual procedures that accompanied most of his treatments. And they also revealed their fear that, in light of current cultural changes, their knowledge would not endure in future generations.

Arafan Sané earned recognition for his ability to cure intestinal problems, as well as female reproductive health, and his reputation was based on the fact that he could cure female infertility. One of the main plants he used was the n'tak (Bridelia micrantha). Its root was collected, scraped and cut into pieces, which were placed in a pot (Fig. 2). The first pieces placed in this manner should add up to seven and were accompanied by a prayer. The preparation of the medicine consisted in boiling; it was taken by the ill every day in a small gourd. Treatment could last for a month or more, depending on the progress of the patient's condition.

Such medicines must be prepared at home to be efficient, because their strength and efficacy lies in their having been supplied to humans by supernatural beings. Phytotherapeutic knowledge belongs to a given lineage, who obtained it in exchange with the supernatural beings (iráns).

The first inhabitants of a region, the founders or socalled "land owners", gained political dominance by having been the first to inhabit a determined area. However,

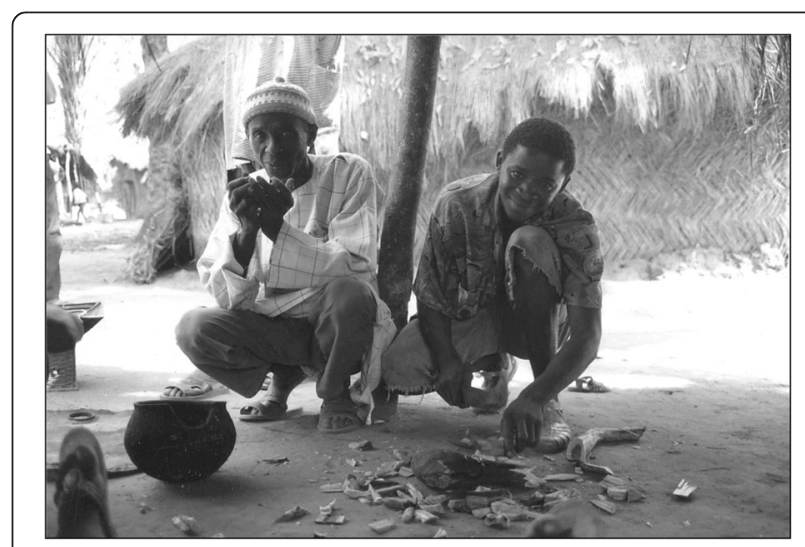

Fig. 2 The healer Arafan Sané and his son/apprentice (lafai Sané) preparing medicine with the root of Bridelia micrantha 
this political dominance is ideologically legitimized by a process of symbolic appropriation of the space, resulting from the imaginary idea that they had accomplished an initial exchange with the supernatural beings [31, 32].

The accounts of initial exchange coincide entirely according to the oldest men:

"The founders took the land from the irán's hands, offering a male nephew who has still not known any woman, and a female niece already full breasted, but who has not yet known any man".

In exchange, they received protection of the supernatural beings - protection for men and for the natural space. They were also given knowledge of plants:

"Household remedies were handed down by the iráns. Our ancestors asked them for plants to cure themselves and their families. These plants are dangerous because ancestors payed dearly for them; in that time they bought much beverage and killed much game. If you start picking these plants without paying for them, you are starting a war with the iráns." (Arafan Sané)

According to this account, the iráns would have lived in the territory founded by the lineage ancestors; they were entities in whose name the healer worked to make his treatments produce the desired effects. This healer was supported by seven iráns. To explain this he used a drawing (Fig. 3) where they are shown standing around the "pots laid out for them to drink".

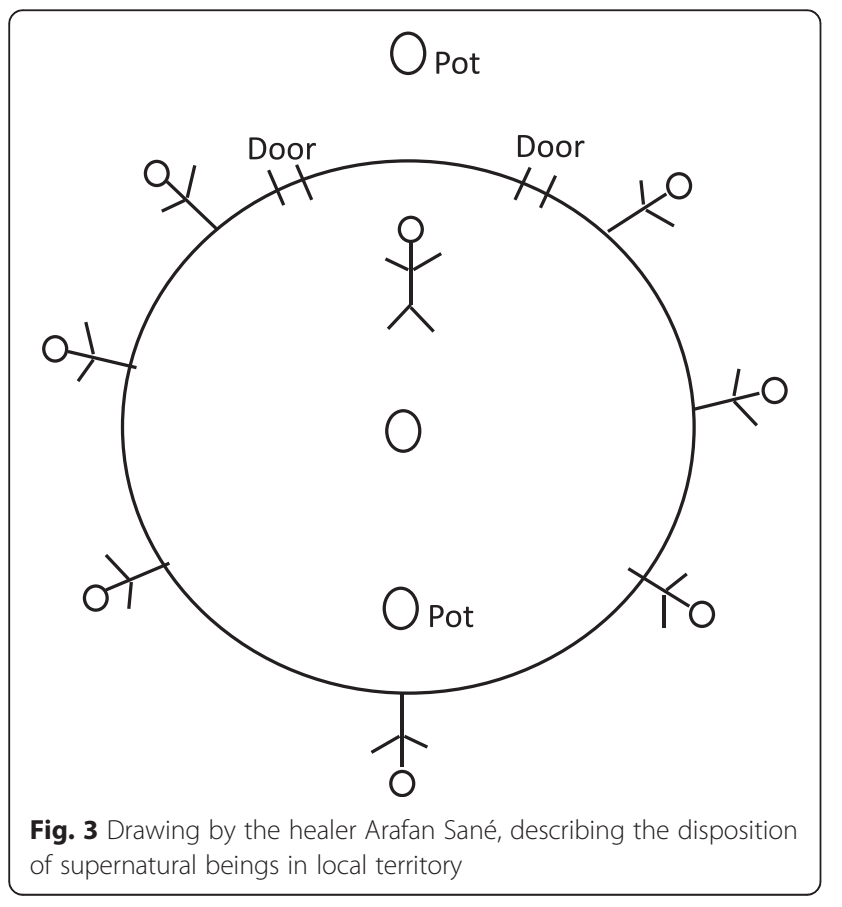

The figures he started drawing represent the iráns who protect the territory. The most important irán, Baken Kantchadáber, was the one with whom Arafan's ancestors did the mythical initial trade, and who lives near the neighboring village of Caontchinque, in a tree known as n'kinan (unidentified). It is he who presided to the nalu's masculine initiation rite (before Islamization), and continues to embody a talisman (cotton yarn) protecting people from the evils that may arise within Nalu territory. Around Baken Kantchadáber stood the "Guardian iráns": Mangbenkar, his son, in the Canábene forest by the river, living in a small tree $n^{\prime}$ koia, (Cf. Synsepalum pobeguinianum) where a large snake or swarm of bees are often seen; Banam, near the village of Arafan (Sogobol), in a huge n'kauuê (kapok; Ceiba pentandra); N'taxanból, in an orchard near the village of Sogobol, in a n'tisebá tree (Pterocarpus santalinoides); Iaulin, near the village of Catomboi, in a termite mound; Kondor, in the area of Iembérem, in a large n'kauuê; and Tukudu, also in Iembérem, in a large n'bim (Dialium guineense) ${ }^{3}$. Just as in human society, Mangbenkar would have occupied his father's place, given the latter's loss of interest in the position due to old age. The two doors represent the openings through which the iráns make their entrance. In this drawing, the figure represented inside the circle would be Mangbenkar. When the healer was on a journey to treat sick people in other villages, he would summon the resident iráns in these areas. For example, when he went to Iembérem, he called for Kondor and Tukudu's help. In his words, he "worked with both of them".

The power of "household remedies" therefore resulted from the efficiency invested on them by supernatural entities, in a process through which the healers possess the knowledge of their ancestors, but derive their power from the genies ${ }^{4}$.

In his turn, Tcherno Camará was a nurse in a Health center in the region, and at the same time was a healer, an agent of traditional medicine. Thus, he was simultaneously an agent of two distinct medical systems. He learned to cure with plants from his grandfather, an expert djambakus; and trained to be a nurse in a public nursing course taken after Guinea-Bissau's independence (1974). The knowledge of modern medicine that he brought to his healer activity was mainly in terms of Portuguese disease terminology. Patients appeared at his doorstep, mostly seeking a cure for malaria, venereal diseases, "belly" aches or eye diseases. Men also commonly brought up impotence problems, while women sought a cure for lack of menstruation and infertility. To solve all these conditions, he resorted to a wide range of plants (some of which are described in Table 1).

Arafan and Tcherno worked in the name of the supernatural beings, so that the plants would achieve the desired ends, so that the iráns should grant him efficacy. 
Their skills as healers derived from the symbolic power consolidated from their ancestors.

Intervention by the iráns is associated with the presence of ritual objects that have become known as the sculptures of the Nalu people [31,33]. One of these, designated as Numba (or Nimba), is a sculpture which may assume two different shapes: one resembles the bust of a woman, and the other - which is predominant in the region in question - resembling a turtle, whose function is to protect houses and their inhabitants, with their power also bearing influence on the fertility of women and fields. The other, called N'tomon (also called Matchol in other regions, the name by which this sculpture is known in African Art markets), has the appearance of a stylized bird, and is connected with pre-Islamic rituals used to solve matters of justice, requests for individual blessings (such as wealth or descent) and also governs matters connected with the fate of lineages.

These healers were also ritual masters, and therefore the efficiency of their treatments was not based solely on their knowledge of the features of vegetable medicines, but also on the strength that N'tomon conferred upon them. Many of these sculptures disappeared with colonization, some of them being initially appropriated by European museums or African Art markets, and later losing their importance with Islamization. Unlike Tcherno, who owned several N'tomon sculptures, in Arafan's village there no longer existed any sculptures. Nevertheless, he maintained the rituals of preIslamic origin that conferred symbolical efficiency to his treatments, even carrying them out on the site where a sculpture had previously stood. Today, both of their children continue their work. Arafan's son, Iafai Sané, uses his knowledge of medicinal plants learned from his father in the treatment of patients from neighboring villages. Given that he was still young when his father died - thus not having completed his initiation in Nalu ritual proceedings, and consequently not having mastered all the secrets -, he is only fit to perform part of the ritual tasks. Tcherno's son, Amará Camará, substitutes his father completely, becoming locally acknowledged healer and ritual master.

The religious transformations inherent to the growing importance of Islam, and the cultural changes implied by the modernization of Guinea-Bissau's economic standards, have marked the life of the Nalu over the last decades [24, $34,35]$, leading to the disappearance of ritual objects and the beliefs attached to them. However, the continuance of these healers' work and knowledge through their sons shows that cultural shifts are not operated abruptly, but in a process of resilience rather than rupture.

\section{The power of sacred writing on plants - marabout Dgibi Camará}

As a result of Islamization, the use and the symbolic perception of plants has become distinct from pre-Islamic perception. However, these two different worldviews coexist, and the infirm often follow parallel therapeutic courses, resorting simultaneously to pre-Islamic medicine healers and marabouts, as well as to the local nurses working for the healthcare system. In other words, "the whole symbolical and therapeutic range is used to ensure effectiveness of the cure. The different axioms are not perceived as contradictory, and the only governing rationale is that of the cure" ([16], p. 115). On the other hand, the syncretic nature of African Islam (e.g. [36, 37]) leads to a crossing between supernatural entities and conceptions of natural elements.

Marabouts perform different activities. They are educated in the complex Islamic mystical system; in numerology and astronomy (e.g. [38-41]). They act as healers, combining a therapeutic based on medicinal plants, with divination and Islamic prayer. Their therapeutic activity is thus combined with magical activity, although just like other local medicine agents they become specialized in the treatment of certain diseases, particularly those with a magic-religious etiology. But the activity of marabouts can also contain an element of negative magic, since they are trained to cast curses, including diseases.

The medical practices of these marabouts differ from those of other healers, not just because the treatments are always accompanied by Quranic prayers, but also because the medicines used in many of them are prepared from vegetable elements mixed with nás (sacred water obtained by washing a wooden tablet on which passages of the Quran are inscribed with vegetable pigments) ${ }^{5}$.

Dgibi Camará underwent a long Quranic education, and his use of medicinal plants concurs with the Muslim practice system. He was the pupil of a Muslim master from a different ethnicity (Djacanca), from whom he learned the Islamic writing, religion and magic, as well as the uses of many medicinal plants. Nevertheless, his knowledge was completed with extensive teachings in traditional medicine from an old Nalu healer.

This marabout has some simple uses for medicinal plants that don't imply ritual procedures. N'sansan (Cassia sieberiana), for instance, is used for body aches. However, most medicines are prepared with nás. As he explained:

“A medicine is mixed with God's name, with the Quran's name; the nás is made, given to the sick person, who then makes an infusion with the medicine: it is boiled and the nás is added, and then it is drunk. It is like when you cook rice on which you put the garnish. The medicine is the rice, and the nás is the garnish. If you cook only the rice, you can eat it but it has no flavor, but when you add the nás, the medicine increases its power."

Thus, the nás increases the power of n'bukui bark (Bauhinia thonningii) used, amongst other things, to 
cure hemorrhoids; or n'sópsóp leaves (Annona muricata) used for stomach aches, or even the treatment of swollen limbs (probably Filariasis) using parts of several plants such as n'txamborta (Diospyros heudelotii) and n'fakat (Hibiscus sterculiifolius).

As a marabout, Dgibi is also a specialist in the treatment of diseases with religious or magical causes. In Islamic terms, these diseases are attributed to the intervention of genies (jinn) and setani (satan), the name for devil, as witnessed among other African Muslim societies (e.g. $[42,43])$. The descriptions, both of the symptoms and of the facts that caused them, are all but identical to the reports of the diseases caused by the iráns in the nosology of pre-Islamic origin. These diseases result from the sexual desire of setani or from breaching a contract made with one of these supernatural beings. There are also suitable medicines and nás for these diseases.

Therefore, the perception of diseases is similar amongst healers and marabouts, as well as amongst the sick who seek their services. Nevertheless, with the progressive Islamization there has been, on the one hand, a reinterpretation of non-natural etiologic categories and the substitution of pre-Islamic supernatural entities by Islamic genies $[16,44]$. On the other hand, we have seen an increasing demand for marabouts to the detriment of masters who follow traditional pre-Islamic rituals, especially in the treatment of diseases of magical-religious nature.

\section{Conclusion}

This study demonstrated the importance of symbolic aspects in the use of medicinal plants, agreeing with the conclusions reached by other studies on the treatment of sickness in West Africa, as for example the work by Fainzang, where it is stated that "(...) the therapeutic discourse affirmed the treatment's validity, leading to eventual intervention by the sacred to legitimate it; the cure was more recommended for its magic (symbolic) value than for its intrinsic value" ([45], p. 420).

In the pre-Islamic worldview prevailing within Nalu's traditional phytotherapy system, the concept of medicinal plants is part of a specific cosmology. In general, natural beings, whether animals or plants, are seen as elements of the world at the same level as humans in a holistic and systemic vision of the universe [46]. The different cosmologic elements are understood not as neutral forces, but as vital essences. As in other African contexts the agency of supernatural entities is similarly acknowledged (e.g. $[47,48]$ ). Humans, plants, and supernatural entities (iráns) participate in a complex network of social relations.

It can be stated that amongst the Nalu, medicinal plants occupy a different place in the pre-Islamic medicine and in the medicine practiced by Islamic masters. Knowledge of plants' pharmacological action will be similar in every respect, but they differ in the symbolical means used to render them efficient. The Muslim practitioner seizes divine energy, allowing himself to be penetrated by its supernatural efficiency. It is easy to understand that this quasi-sacramental value conceded to the reading of the Quran has drifted to more practical uses since the first generations of Islam, and that the versicles have come to be used for purposes of healing or divination $[40,49]$. The presence of sacred words in medicine is "a direct way of infusing the body with the sacred liturgy, yet another kind of embodiment of the text and a textualization, hence sanctification, of the body" ([50], p. 27).

In the healing practices based on pre-Islamic conceptions, plants are mediators for the power of iráns. Given that plants with beneficial properties were an offering from the iráns to the forefathers, plants themselves possess a magical and symbolical power. Plants' medicinal value stems from a bond established through human lineages, which includes both the living and their ancestors [51], in a line of continuity with the supernatural sphere.

Within Muslim practices, medicinal plants become simply a material instrument, and the magical effect lies with the reproduction of the sacred text, in a direct divine intervention. Although the marabout's power is also mediated by the strength of Islamic genies, these do not possess the autonomy of pre-Islamic genies. Thus, it is as if the material means found in the vegetable elements had lost part of their symbolic meaning and value to the power of the new medium afforded by the sacred writings ${ }^{6}$.

In conclusion, in the Nalu healing systems under analysis, we found a similar degree of knowledge and use of medicinal plants amongst healers who follow a non-Islamic cosmology and healers who perform Islamic ritualistic practices. Distinctions in the use of medicinal plants are drawn according to the healers' field of expertise, gender, personal history and individual learning careers. Nevertheless, the non-Muslim and Muslim procedures involved in the bestowal of symbolic efficacy on treatments differ and are supported by distinct ontologies: to the former, plants possess an intrinsic value derived from the bonds linking human and superhuman entities, while for the latter plants relinquish that value to the relation established directly by humans with God, mediated by Holy Scriptures.

\section{Endnotes}

${ }^{1}$ Other studies report the traditional medical systems (notions of disease, treatments and diagnostics) without any reference to medicinal plants, of Manjack $[47,52]$ and Felupe/Djola people [53].

${ }^{2}$ In fact, Hajia Bintu Camarás specialty is locally known as midwifery, and not exactly as a healer. Her medicinal practices and the social recognition that her knowledge received were only revealed to me through daily observations, which explains their inclusion in this study. 
${ }^{3}$ The sites where iráns live are considered sacred and as such are preserved. These sacred forests have recently been considered protected areas, in a process that can be described as a reinterpretation of their meaning or as a commodification of nature [46].

${ }^{4}$ Although the power of healers derives from the genies, like in other African cultures e.g. [54], there is no possession ritual.

${ }^{5}$ The use of dissolved written Quran verses in water as preventive or curative treatments is common in many Islamic contexts (e.g. [41, 50, 55]).

${ }^{6}$ In other African contexts marked by the expansion of the African Independent Churches, we can witness a similar process. Even though the agents of these churches may possess a thorough knowledge of medicinal plants equal to traditional healers [56], their understanding of the curing processes are not concordant, giving rise to a conflict between the treatments performed by traditional healers and the treatments performed in these churches by their prophets or pastors. For instance, in South Africa and Mozambique, the Zionist $[57,58]$, and Pentecostal churches $[59,60]$ reject the influence of the spirits from traditional African religions on the curing process, the spiritual dimension of the treatment being transferred to the Holy Spirit, through biblical texts and prayers.

\section{Acknowledgements}

I wish to thank all the healers - Arafan Sané, Hajia Bintu Camará, Dgibi Camará, Fatu Bangorá, Usumane Cassamá, and Tcherno Camará - for their hospitality, generosity and sharing of knowledge, and Abu Camará for his friendship and support in field tasks. I am also grateful to my colleagues Maria Cardeira da Silva and José Mapril that helped me find the literature concerning magic uses of Islamic texts.

This work would not have been possible without the contribution in identifying the botanical material of the IICT (Tropical Research Institute, Lisbon) research team - Maria Adélia Diniz, Eurico Martins, Fernanda Pinto-Bastos, and Luís Catarino.

\section{Funding}

This article was funded by FCT (Fundação Ciência e Tecnologia, Portugal) through UID/ANT/04038/2013. The field missions were supported through two individual research grants (Calouste Gulbenkian Foundation; JNICTJunta Nacional para a Investigação Científica e Tecnológica, Portugal/Praxis XXI) and the project "Etno-botânica. Usos e concepções das plantas no mato de Cantanhez" (NGO AD - Acção para o Desenvolvimento, Guinea-Bissau, funded by IUCN), and through two research projects of CRIA (Centre for Research in Anthropology, Portugal) - POCI/ANT/57434/2004 and PTDC/CSANT/121124/2010, funded by FCT.

\section{Authors' contributions}

The author is responsible for all parts of this paper, such as data collection and analysis, and writing of the manuscript.

\section{Authors' information}

This article is in memoriam of Arafan Sané.

\section{Competing interests}

The author declares that she has no competing interests.

Received: 15 January 2016 Accepted: 19 May 2016

Published online: 17 June 2016

\section{References}

1. World Bank. 2014. http://www.worldbank.org/en/country/guineabissau/ overview. Acessed 20 July 2015.

2. World Health Organization. Guinea-Bissau: WHO statistical profile. 2015. http://www.who.int/gho/countries/gnb.pdf?ua=1. Acessed 19 July 2015.

3. Diniz MA, Silva O, Paulo MA, Gomes ET. Medicinal uses of plants from GuineaBissau. In: Van Der Maesen LJG, Van Der Burgt XM, Van Medenbach De Rooy JM, editors. The biodiversity of African plants. Proceedings of the XIVth AETFAT Congress. Dordrecht: Kluwer Academic Publishers; 1996. p. 727-31.

4. Indjai B, Catarino L, Mourão D. Mezinhos de Orango - Plantas Medicinais e Pessoas da Ilha da Rainha Pampa. Bissau: Instituto da Biodiversidade e das Áreas Protegidas; 2010.

5. Indjai B, Barbosa C, Catarino L. Mezinhos da terra e curas tradicionais nas Ilhas de Canhabaque e do Parque Nacional Marinho João Vieira e Polião. Instituto da Biodiversidade e das Áreas Protegidas: Bissau; 2014.

6. Diniz MA, Martins ES, Silva O, Gomes E. Contribuição para o conhecimento de plantas medicinais da Guiné-Bissau. Portugaliae Acta Biológica. 2000;19:417-27.

7. Gomes E, Silva OM, Diniz MA, Martins ES. Plantas Medicinais da Guiné-Bissau - Manual Prático. Bissau: Acção para o Desenvolvimento; 2003.

8. Martins AS, Gomes ET, Silva O. Caracterização botânica da liana medicinal da Guiné-Bissau Calycobolus heudelotii. In: Roque AC, Torrão MM, Havik P, editors. Actas do Workshop Plantas Medicinais e Práticas Fitoterapêuticas nos Trópicos (CD-Rom). Lisboa: Instituto de Investigação Cientifica Tropical; 2009.

9. Romeiras MM, Duarte MC, Indjai B, Catarino L. Medicinal Plants Used to Treat Neurological Disorders in West Africa: A Case Study with GuineaBissau Flora. Am J Plant Sci. 2012;3:1028-36. doi:10.4236/ajps.2012.327122.

10. Silva O, Duarte A, Cabrita J, Pimentel M, Diniz A, Gomes E. Antimicrobial activity of Guinea-Bissau traditional remedies. J Ethnopharmacol. 1996;50(1):55-9.

11. Sousa MR, Silva O, Gomes ET, Poiares-da-Silva J. Antigiardial activity of Guinea-Bissau's medicinal plants. Clin Microbiol Infect. 2001;7(1):244.

12. Etkin NL. Ethnopharmocology: biobehavioral approaches in the anthropological study of indigenous medicines. Annu Rev Anthropol. 1988;17:23-42.

13. Ingold T. Two reflections on ecological knowledge. In: Sanga G, Ortalli G, editors. Nature Knowledge. Ethnoscience, Cognition, and Utility. New York: Berghahn Books; 2003. p. 301-11.

14. Etkin N. Cultural constructions of efficacy. In: Van der Geest S, Whyte RS, editors. The context of medicines in developing countries. Dordrecht: Kluwer Academic Publishers; 1988. p. 299-326.

15. Augé M. Ordre biologique, ordre social: la maladie forme élémentaire de l'événement. In: Augé M, Herzlich C, editors. Le Sens du Mal. Anthropologie, Histoire, Sociologie de la Maladie. Paris: Editions des Archives Contemporaines; 1984. p. 35-81.

16. Fassin D. Pouvoir et maladie en Afrique. Paris: PUF; 1992.

17. Feierman S, Janzen JM. The social basis of health and healing in Africa. Berkeley: University of California Press; 1992

18. Janzen JM, Green EC. Continuity, change, and challenge in African medicine. In: Selin H, editor. Medicine across cultures: history and practice of medicine in non-western cultures. Dordrecht: Kluwer Academic Publishers; 2003. p. 1-26.

19. Quiroz D, van Andel T. Evidence of a link between taboos and sacrifices and resource scarcity of ritual plants. J Ethnobiol Ethnomed. 2015;11:5.

20. Gruca M, van Andel TR, Balslev H. Ritual use of palms in traditional medicine in sub-Saharan Africa: a review. J Ethnobiol Ethnomed. 2014;10:60.

21. Frazão-Moreira A. A natureza em perspectiva: reflexões sobre saberes ecológicos locais e conhecimentos científicos. In: Alves A, Souto F, Peroni N, editors. Etnoecologia em perspectiva: natureza, cultura e conservação. Recife: NUPEEA; 2010. p. 73-88.

22. Gonçalves J. O Mundo Árabo-Islâmico e o Ultramar Português. Lisboa: Junta de Investigação do Ultramar; 1958.

23. Carreira A. Organização social e económica dos povos da Guiné Portuguesa. Boletim Cultural da Guiné Portuguesa. 1961;16I(64):641-736.

24. Frazão-Moreira A. Plantas e "Pecadores". Percepções da Natureza em África. Lisboa: Livros Horizonte; 2009.

25. Alexiades M, editor. Selected Guidelines for Ethnobotanical Research: A Field Manual. New York: NYBC; 1996.

26. Bernard HR. Research Methods in Anthropology. Qualitative and Quantitative Approaches. Walnut Creek: Altamira Press; 1995.

27. Frazão-Moreira A. Usos e concepções das plantas no mato de Cantanhez. Relatório Iniciativa de Cantanhez. Bissau: Acção para o Desenvolvimento; 1995.

28. Hepper FN, editor. Flora of West Tropical Africa. London: Crown Agents for Oversea Governments and Administrations; 1968. 
29. Madden R. Being Ethnographic: A Guide to the Theory and Practice of Ethnography. London: Sage; 2010

30. Sindzingre N. La nécessité du sens: l'explication de l'infortune chez les senufo. In: Augé M, Herzlich C, editors. Le Sens du Mal. Paris: Editions des Archives Contemporaines; 1984. p. 93-122.

31. Curtis MY. À la rencontre des Nalu. Arts de la côte de Guinée. Paris: I'Harmatta; 2013.

32. Frazão-Moreira A. Récits de migration entre les nalou de Cubucaré. In: Gaillard G, editor. Migrations anciennes et peuplement actuel des basses cotes guinéennes. Paris: Harmattan; 2000. p. 403-12.

33. Stocchero D. Cosmologia Nalu: una ricostruzione etnologica fra passato e presente. Antrocom. 2005;1(1):5-15.

34. Lundy B. Ethnic Encounters and Everyday Economics in Kassumba, GuineaBissau. Ethnopolitics. 2012;11(3):235-54.

35. Temudo MP. A narrativa da degradação ambiental no Sul da Guiné-Bissau: uma desconstrução etnográfica. Etnográfica. 2009;13(2):237-64.

36. Trimingham JS. The Influence of Islam upon Africa. New York: Longham; 1980.

37. Lewis IM, editor. Islam in tropical Africa. Bloomington \& London: International African Institute; 1966

38. La LE. Démonologie. Paris: Payot; 1951.

39. Chelhod J. Les Structures du Sacré Chez les Arabes. Paris: Maisonneuve et Larose; 1986.

40. Lory P. Verbe coranique et magie en terre d'Islam. Systèmes de Pensée en Afrique. 1993;12:173-86.

41. Bledsoe C, Rovey K. Arabic literacy and secrecy among the mende of sierra Leone. In: Street B, editor. Cross-Cultural Approaches to Literacy. Cambridge: Cambridge University Press; 1997. p. 110-34.

42. Greenwood B. Cold or Spirits? Ambiguity and Syncretism in Moroccan Therapeutics. In: Feierman S, Janzen JM, editors. The social basis of health and healing in Africa. Berkeley: University of California Press; 1992. p. 285-314.

43. Ally Y, Laher S. South African Muslim faith healers perceptions of mental illness: Understanding, aetiology and treatment. J Relig Health. 2008:47(1):45-56.

44. Blanchy C, Lanchy S, Cheikh M, Said M, Allaoui M, Issihka M. Thérapies Traditionnelles aux Comores. Cahiers Sci Humaines. 1993;29(4):763-90

45. Fainzang S. La cure comme mythe: le traitement de la maladie et son idéologie à partir de quelques exemples Ouest-Africains. Cahiers ORSTOM. 1981;18(4):415-21.

46. Frazão-Moreira A. Ethnobiological research and ethnographic challenges in the 'ecological era'. Etnográfica. 2015;19(3):605-24.

47. Crowley EL. Regional spirit shrines and ethnic relations in Guinea-Bissau. Afr J. 1998;17:27-39.

48. Quiroz D. Do not fear the supernatural!: the relevance of ritual plant use for traditional culture, nature conservation, and human health in western Africa, PhD Thesis. Wageningen: Wageningen University; 2015.

49. Goody J. Restricted literacy in Northern Ghana. In: Goody J, editor. Literacy in Traditional Societies. Cambridge: Cambridge University Press; 1981. p. 198-265.

50. Lambek M. Certain knowledge, contestable authority: power and practice on the Islamic periphery. Am Ethnol. 1990;17(1):23-40.

51. Kopytoff I. Ancestors as Elders in Africa. Africa. 1971:41(2):129-42.

52. Crowley E, Ribeiro R. Sobre a medicina tradicional e forma da sua colaboração com a medicina moderna. Soronda. 1987;4:95-110.

53. Costa LMN. Ventos do mal: sopro da cura: cosmovisão, doença e cura entre os Felupes da Guiné-Bissau. Antropologia Portuquesa. 2012;29:25-48.

54. Fainzang S. L'Intérieur des Choses. Maladie, Divination et Reproduction Sociale Chez les Bisa du Burkina. Paris: Harmattan; 1986

55. El-Tom AO. Drinking the Quran: the meaning of Quranic verses in Berti erasure. Africa. 1995:55(4):414-31.

56. Bruschi P, Morganti M, Mancini M, Signorini MA. Traditional healers and laypeople: a qualitative and quantitative approach to local knowledge on medicinal plants in Muda (Mozambique). J Ethnopharmacol. 2011;138(2): 543-63.

57. Kieman JP. The Herder and the Rustler: Deciphering the Affinity Between Zulu Diviner and Zionist Prophet. Afr Stud. 1992;51(1):231-41.

58. Honwana A. Espíritos Vivos, Tradições Modernas. Possessão de Espíritos e Reintegração Social Pós-Guerra no Sul de Moçambique. Lisboa: Ela por Ela; 2003.

59. Pfeiffer J. Commodity Fetichismo, the Holy Spirit, and the turn to Pentecostal and African Independent Churches in Central Mozambique. Culture, Medicine, and. Psychiatry. 2005;39(3):255-83.

60. Pfeiffer J, Gimbel-Sherr K, Augusto OJ. The holy spirit in the household: Pentecostalism, gender, and neoliberalism in Mozambique. Am Anthropol. 2007;109(4):688-700

\section{Submit your next manuscript to BioMed Central and we will help you at every step:}

- We accept pre-submission inquiries

- Our selector tool helps you to find the most relevant journal

- We provide round the clock customer support

- Convenient online submission

- Thorough peer review

- Inclusion in PubMed and all major indexing services

- Maximum visibility for your research

Submit your manuscript at www.biomedcentral.com/submit

) Biomed Central 